\title{
A general class of periodic boundary value problems for controlled nonlinear impulsive evolution equations on Banach spaces
}

\author{
Said Melliani", Abdelati El Allaoui and Lalla Saadia Chadli
}

\section{"Correspondence:}

said.melliani@gmail.com

Laboratoire de Mathématiques

Appliquées \& Calcul Scientifique,

Sultan Moulay Slimane University,

BP 523, Beni Mellal, 23000, Morocco

\begin{abstract}
This paper deals with the periodic boundary value problems for nonlinear impulsive evolution equations with controls. By using the theory of semigroup and fixed point methods, we address some conditions ensuring the existence and uniqueness. Finally, two examples are provided to prove the effectiveness of the proposed results.
\end{abstract}

MSC: 34G10; 34B37; 93C15

Keywords: impulsive evolution equations; periodic boundary value problems; control; mild solution

\section{Introduction}

The theory of impulsive differential equations has lately years been an object of increasing interest because of its vast applicability in several fields including mechanics, electrical engineering, biology, medicine, and so on. Therefore, it has drawn wide attention of the researchers in the recent years, among them we find JinRong Wang, Michal Feckan, Yong Zhou, and others [1-11].

For a wide bibliography and exposition on differential equations with impulses, see for instance [12-18], and there are many papers discussing the impulsive differential equations and impulsive optimal controls with the classic initial condition: $x(0)=x_{0}$ (see [19-24]).

In this paper, we consider the following problems for nonlinear impulsive evolution equations with periodic boundary value:

(IEE)

$$
\left\{\begin{array}{l}
u^{\prime}(t)=A u(t)+f(t, u(t), u(\rho(t)))+B(t) c(t) \\
\quad t \in\left(s_{i}, t_{i+1}\right], i=0,1,2, \ldots, m, c \in \mathcal{U}_{\mathrm{ad}} \\
u(t)=T\left(t-t_{i}\right) g_{i}(t, u(t)), \quad t \in\left(t_{i}, s_{i}\right], i=1,2, \ldots, m \\
u(0)=u(a) \in X
\end{array}\right.
$$

The operator $A: D(A): X \longrightarrow X$ is the generator of a strongly continuous semigroup $\{T(t), t \geq 0\}$ on a Banach space $X$ with a norm $\|\cdot\|$, and the fixed points $s_{i}$ and $t_{i}$ satisfying

$$
0=s_{0}<t_{1} \leq s_{1} \leq t_{2}<\cdots<t_{m} \leq s_{m} \leq t_{m+1}=a
$$

\section{Springer}


are pre-fixed numbers, $f:[0, a] \times X \times X \longrightarrow X$ is continuous, $\rho:[0, a] \longrightarrow[0, a]$ is continuous, and $g_{i}:\left[t_{i}, s_{i}\right] \times X \longrightarrow X$ is continuous for all $i=1,2, \ldots, m$.

\section{Preliminaries}

Next, we review some basic concepts, notations, and technical results that are necessary in our study.

Throughout this paper, $I=[0, a], \mathcal{C}(I, X)$ is the Banach space of all continuous functions from $I$ into $X$ with the norm $\|u\|_{\mathcal{C}}=\sup _{t \in I}\{\|u(t)\|: t \in I\}$ for $u \in \mathcal{C}(I, X)$, and we consider the space

$$
\begin{aligned}
\mathcal{P C}(I, X)= & \left\{u: I \longrightarrow X: u \in \mathcal{C}\left(\left(t_{i}, t_{i+1}\right], X\right), i=0,1, \ldots, m\right. \\
& \text { and there exist } \left.u\left(t_{i}^{-}\right) \text {and } u\left(t_{i}^{+}\right), i=1, \ldots, m \text { with } u\left(t_{i}^{-}\right)=u\left(t_{i}\right)\right\},
\end{aligned}
$$

endowed with the Chebyshev PC-norm $\|u\|_{\mathcal{P C}}=\sup _{t \in I}\{\|u(t)\|: t \in I\}$ for $u \in \mathcal{P C}(I, X)$. Denote $M=\sup _{t \in I}\|T(t)\|$.

Let $Y$ be another separable reflexive Banach space where the controls $c$ take values. Denote by $P_{f}(Y)$ a class of nonempty closed and convex subsets of $Y$. We suppose that the multivalued map $w:[0, T] \longrightarrow P_{f}(Y)$ is measurable, $w(\cdot) \subset E$, where $E$ is a bounded set of $Y$, and the admissible control set

$$
\mathcal{U}_{\mathrm{ad}}=\left\{c \in L^{p}(E): c(t) \in w(t) \text {, a.e. }\right\}, \quad p>1 .
$$

Then $\mathcal{U}_{\mathrm{ad}} \neq \emptyset$, which can be found in [25]. Some of our results are proved using the next well-known results.

Theorem 1 (Krasnoselskii's fixed point theorem) Assume that $K$ is a closed bounded convex subset of a Banach space X. Furthermore assume that $\Gamma_{1}$ and $\Gamma_{2}$ are mappings from $K$ into $X$ such that:

1. $\Gamma_{1}(u)+\Gamma_{2}(v) \in K$ for all $u, v \in K$,

2. $\Gamma_{1}$ is a contraction,

3. $\Gamma_{2}$ is continuous and compact.

Then $\Gamma_{1}+\Gamma_{2}$ has a fixed point in $K$.

To begin our discussion, we need to introduce the concept of a mild solution for (IEE). Assume that $u:[0, a] \longrightarrow X$ is a solution of

$$
u^{\prime}(t)=A u(t)+f(t, u(t), u(\rho(t)))+B(t) c(t), \quad 0 \leq t \leq a .
$$

From the theory of strongly continuous semigroups, we get

$$
\begin{aligned}
u(t) & =T(t) u(0)+\int_{0}^{t} T(t-s)(f(s, u(s), u(\rho(s)))+B(s) c(s)) d s \\
& =T(t) u(a)+\int_{0}^{t} T(t-s)(f(s, u(s), u(\rho(s)))+B(s) c(s)) d s \\
& =T(t)\left[T\left(a-t_{m}\right) g_{m}\left(s_{m}, u\left(s_{m}\right)\right)\right.
\end{aligned}
$$




$$
\begin{aligned}
& \left.+\int_{s_{m}}^{a} T(a-s)(f(s, u(s), u(\rho(s)))+B(s) c(s)) d s\right] \\
& +\int_{0}^{t} T(t-s)(f(s, u(s), u(\rho(s)))+B(s) c(s)) d s \quad \text { for all } t \in\left[0, t_{1}\right]
\end{aligned}
$$

and

$$
\begin{aligned}
u(t) & =T\left(t-s_{i}\right) u\left(s_{i}\right)+\int_{s_{i}}^{t} T(t-s)(f(s, u(s), u(\rho(s)))+B(s) c(s)) d s \\
& =T\left(t-s_{i}\right) T\left(s_{i}-t_{i}\right) g_{i}\left(s_{i}, u\left(s_{i}\right)\right)+\int_{s_{i}}^{t} T(t-s)(f(s, u(s), u(\rho(s)))+B(s) c(s)) d s \\
& =T\left(t-t_{i}\right) g_{i}\left(s_{i}, u\left(s_{i}\right)\right)+\int_{s_{i}}^{t} T(t-s)(f(s, u(s), u(\rho(s)))+B(s) c(s)) d s
\end{aligned}
$$

for all $t \in\left(s_{i}, t_{i+1}\right], i=1,2, \ldots, m$.

This expression motivates the following definition.

Definition 1 We say that a function $u \in \mathcal{P C}(I, X)$ is called a mild solution of the problem (IEE), if $u$ satisfies

$$
\left\{\begin{aligned}
u(t)= & T(t)\left[T\left(a-t_{m}\right) g_{m}\left(s_{m}, u\left(s_{m}\right)\right)+\int_{s_{m}}^{a} T(a-s)(f(s, u(s), u(\rho(s)))+B(s) c(s)) d s\right] \\
& +\int_{0}^{t} T(t-s)(f(s, u(s), u(\rho(s)))+B(s) c(s)) d s, \quad t \in\left[0, t_{1}\right] \\
u(t)= & T\left(t-t_{i}\right) g_{i}(t, u(t)), \quad t \in\left(t_{i}, s_{i}\right], i=1,2, \ldots, m \\
u(t)= & T\left(t-t_{i}\right) g_{i}\left(s_{i}, u\left(s_{i}\right)\right)+\int_{s_{i}}^{t} T(t-s)(f(s, u(s), u(\rho(s)))+B(s) c(s)) d s \\
t \in & \left(s_{i}, t_{i+1}\right], i=1,2, \ldots, m .
\end{aligned}\right.
$$

\section{Existence and uniqueness of mild solutions}

To establish our results, we introduce the following assumptions:

$\left(\mathrm{H}_{0}\right) \quad$ 1. $A: D(A) \subseteq X \rightarrow X$ is the generator of a strongly continuous semigroup $\{T(t), t \geq 0\}$ on $X$ with a norm $\|\cdot\|$.

2. $B:[0, a] \longrightarrow \mathcal{L}(Y, X)$ is essentially bounded, i.e., $B \in L^{\infty}([0, a], \mathcal{L}(Y, X))$.

$\left(\mathrm{H}_{1}\right)$ We have the functions $f \in \mathcal{C}(I \times X \times X, X), g_{i} \in \mathcal{C}\left(\left[t_{i}, s_{i}\right] \times X, X\right), i=1,2, \ldots, m$, and $\rho: I \longrightarrow I$ is continuous.

$\left(\mathrm{H}_{2}\right)$ There is a constant $C_{f}, L_{f}>0$ such that

$$
\left\|f\left(t, u_{1}, v_{1}\right)-f\left(t, u_{2}, v_{2}\right)\right\| \leq C_{f}\left\|u_{1}-u_{2}\right\|+L_{f}\left\|v_{1}-v_{2}\right\|
$$

for each $t \in\left[s_{i}, t_{i+1}\right], u_{1}, u_{2}, v_{1}, v_{2} \in X$ and $i=0,1, \ldots, m$.

$\left(\mathrm{H}_{3}\right)$ There is a constant $L>0$ such that

$$
\|f(t, u, v)\| \leq L\left(1+\|u\|^{\mu}+\|v\|^{v}\right)
$$

for all $t \in\left[s_{i}, t_{i+1}\right]$ and all $u, v \in X, i=0,1, \ldots, m$, and $\mu, v \in[0,1]$.

$\left(\mathrm{H}_{4}\right)$ There is a constant $C_{g_{i}}>0, i=1,2, \ldots, m$, such that

$$
\left\|g_{i}(t, u)-g_{i}(t, v)\right\| \leq C_{g_{i}}\|u-v\|
$$

for each $t \in\left[t_{i}, s_{i}\right]$, and all $u, v \in E^{n}, i=1,2, \ldots, m$. 
$\left(\mathrm{H}_{5}\right)$ There is a function $t \longmapsto \psi_{i}(t), i=1,2, \ldots, m$, such that

$$
\left\|g_{i}(t, u)\right\| \leq \psi_{i}(t)
$$

for each $t \in\left[t_{i}, s_{i}\right]$ and all $u \in X$.

We put $C=\max _{1 \leq i \leq m} C_{g_{i}}$ and $N_{i}=\sup _{t \in\left[t_{i}, s_{i}\right]} \psi_{i}(t)<+\infty$.

Remark 1 From the assumptions $\left(\mathrm{H}_{0}\right)-\left(\mathrm{H}_{2}\right)$ and the definition of $\mathcal{U}_{\text {ad }}$, it is also easy to verify that $B c \in L^{p}([0, a] ; X)$ with $p>1$ for all $c \in \mathcal{U}_{\text {ad }}$.

Therefore, $B c \in L^{1}([0, a] ; X)$ and $\|B c\|_{L^{1}}<\infty$.

Now, we can establish our first existence result.

Theorem 2 Let assumptions $\left(\mathrm{H}_{0}\right),\left(\mathrm{H}_{1}\right),\left(\mathrm{H}_{2}\right)$, and $\left(\mathrm{H}_{4}\right)$ be satisfied. Suppose, in addition, that the following property is verified:

$$
\begin{aligned}
\lambda:= & M \max \left\{\max _{1 \leq i \leq m}\left\{C_{g_{i}}+\left(C_{f}+L_{f}\right)\left(t_{i+1}-s_{i}\right)\right\},\right. \\
& \left.C, M C_{g_{m}}+\left(C_{f}+L_{f}\right) M\left(a-s_{m}\right)+\left(C_{f}+L_{f}\right) t_{1}\right\}<1 .
\end{aligned}
$$

Then the problem (IEE) has a unique mild solution.

Proof Define a mapping $\Gamma: \mathcal{P C}(I, X) \longrightarrow \mathcal{P C}(I, X)$ by

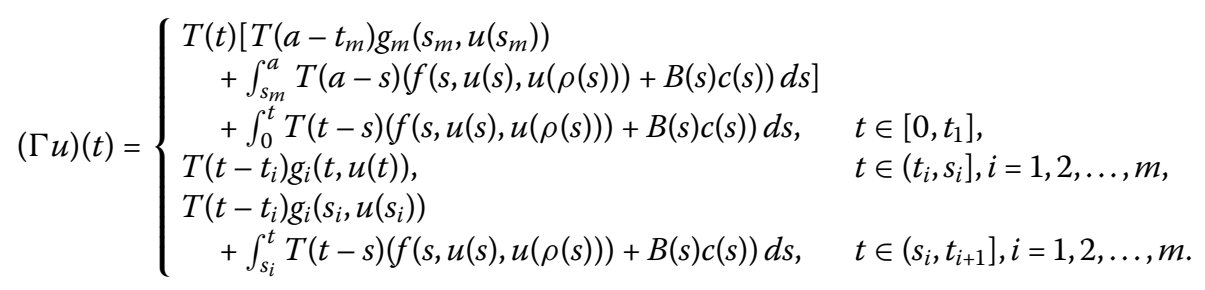

Let $h>0$ be very small and $u \in \mathcal{P C}(I, X)$, we have the following.

Case 1: For $t \in\left[0, t_{1}\right]$, we have

$$
\begin{aligned}
& \|(\Gamma u)(t+h)-(\Gamma u)(t)\| \\
& =\| T(t+h)\left[T\left(a-t_{m}\right) g_{m}\left(s_{m}, u\left(s_{m}\right)\right)+\int_{s_{m}}^{a} T(a-s)(f(s, u(s), u(\rho(s)))+B(s) c(s)) d s\right] \\
& \quad+\int_{0}^{t+h} T(t+h-s)(f(s, u(s), u(\rho(s)))+B(s) c(s)) d s \\
& \quad-T(t)\left[T\left(a-t_{m}\right) g_{m}\left(s_{m}, u\left(s_{m}\right)\right)+\int_{s_{m}}^{a} T(a-s)(f(s, u(s), u(\rho(s)))+B(s) c(s)) d s\right] \\
& \quad-\int_{0}^{t} T(t-s)(f(s, u(s), u(\rho(s)))+B(s) c(s)) d s \| \\
& \leq M \| T(h)\left[T\left(a-t_{m}\right) g_{m}\left(s_{m}, u\left(s_{m}\right)\right)+\int_{s_{m}}^{a} T(a-s)(f(s, u(s), u(\rho(s)))+B(s) c(s)) d s\right] \\
& \quad-\left[T\left(a-t_{m}\right) g_{m}\left(s_{m}, u\left(s_{m}\right)\right)+\int_{s_{m}}^{a} T(a-s)(f(s, u(s), u(\rho(s)))+B(s) c(s)) d s\right] \|
\end{aligned}
$$




$$
\begin{aligned}
& +M \int_{0}^{h}\|f(s, u(s), u(\rho(s)))+B(s) c(s)\| d s+M \int_{0}^{t}\|B(s+h) c(s+h)-B(s) c(s)\| d s \\
& +M \int_{0}^{t}\|f(s+h, u(s+h), u(\rho(s+h)))-f(s, u(s), u(\rho(s)))\| d s \rightarrow 0 \quad \text { as } h \rightarrow 0 .
\end{aligned}
$$

Case 2: For $t \in\left(t_{i}, s_{i}\right], i=1, \ldots, m$, we have

$$
\begin{aligned}
\|(\Gamma u)(t+h)-(\Gamma u)(t)\| & =\left\|T\left(t+h-t_{i}\right) g_{i}(t+h, u(t+h))-T\left(t-t_{i}\right) g_{i}(t, u(t))\right\| \\
& \leq M\left\|T(h) g_{i}(t+h, u(t+h))-g_{i}(t, u(t))\right\| \rightarrow 0 \quad \text { as } h \rightarrow 0 .
\end{aligned}
$$

Case 3: For $t \in\left(s_{i}, t_{i+1}\right], i=1, \ldots, m$, we have

$$
\begin{aligned}
& \|(\Gamma u)(t+h)-(\Gamma u)(t)\| \\
& =\| T\left(t+h-t_{i}\right) g_{i}\left(s_{i}, u\left(s_{i}\right)\right)+\int_{s_{i}}^{t+h} T(t+h-s)(f(s, u(s), u(\rho(s)))+B(s) c(s)) d s \\
& \quad-T\left(t-t_{i}\right) g_{i}\left(s_{i}, u\left(s_{i}\right)\right)-\int_{s_{i}}^{t} T(t-s)(f(s, u(s), u(\rho(s)))+B(s) c(s)) d s \| \\
& \leq M\left\|T(h) g_{i}\left(s_{i}, u\left(s_{i}\right)\right)-g_{i}\left(s_{i}, u\left(s_{i}\right)\right)\right\|+M \int_{s_{i}}^{s_{i}+h}\|f(s, u(s), u(\rho(s)))+B(s) c(s)\| d s \\
& \quad+M \int_{s_{i}}^{t}\|B(s+h) c(s+h)-B(s) c(s)\| d s \\
& \quad+M \int_{s_{i}}^{t}\|f(s+h, u(s+h), u(\rho(s+h)))-f(s, u(s), u(\rho(s)))\| d s \rightarrow 0 \quad \text { as } h \rightarrow 0 .
\end{aligned}
$$

Then $\Gamma$ is well defined and $\Gamma u \in \mathcal{P C}(I, X)$ for all $u \in \mathcal{P C}(I, X)$.

Now we only need to show that $\Gamma$ is a contraction mapping.

Case 1: For $u, v \in \mathcal{P C}(I, X)$ and $t \in\left[0, t_{1}\right]$, we have

$$
\begin{aligned}
\|(\Gamma u)(t)-(\Gamma v)(t)\| & \| T(t)\left[T\left(a-t_{m}\right) g_{m}\left(s_{m}, u\left(s_{m}\right)\right)+\int_{s_{m}}^{a} T(a-s)(f(s, u(s), u(\rho(s)))+B(s) c(s)) d s\right] \\
& +\int_{0}^{t} T(t-s)(f(s, u(s), u(\rho(s)))+B(s) c(s)) d s \\
& -T(t)\left[T\left(a-t_{m}\right) g_{m}\left(s_{m}, v\left(s_{m}\right)\right)+\int_{s_{m}}^{a} T(a-s)(f(s, v(s), v(\rho(s)))+B(s) c(s)) d s\right] \\
& -\int_{0}^{t} T(t-s)(f(s, v(s), v(\rho(s)))+B(s) c(s)) d s \| \\
\leq & M\left[M C_{g_{m}}\left\|u\left(s_{m}\right)-v\left(s_{m}\right)\right\|+M \int_{s_{m}}^{a}\left(C_{f}\|u(s)-v(s)\|+L_{f}\|u(\rho(s))-v(\rho(s))\|\right) d s\right] \\
& +M \int_{0}^{t}\left(C_{f}\|u(s)-v(s)\|+L_{f}\|u(\rho(s))-v(\rho(s))\|\right) d s \\
\leq & M\left[M C_{g_{m}}+\left(C_{f}+L_{f}\right) M\left(a-s_{m}\right)+\left(C_{f}+L_{f}\right) t_{1}\right]\|u-v\|_{\mathcal{P C}} \\
\leq & \lambda\|u-v\|_{\mathcal{P C} .}
\end{aligned}
$$


Case 2: For $u, v \in \mathcal{P C}(I, X)$ and $t \in\left(t_{i}, s_{i}\right], i=1, \ldots, m$, we have

$$
\begin{aligned}
\|(\Gamma u)(t)-(\Gamma v)(t)\| & =\left\|T\left(t-t_{i}\right) g_{i}(t, u(t))-T\left(t-t_{i}\right) g_{i}(t, v(t))\right\| \\
& \leq M C_{g_{i}}\|u-v\|_{\mathcal{P C}} \\
& \leq M C\|u-v\|_{\mathcal{P C}} \\
& \leq \lambda\|u-v\|_{\mathcal{P C}} .
\end{aligned}
$$

Case 3: For $u, v \in \mathcal{P C}(I, X)$ and $t \in\left(s_{i}, t_{i+1}\right], i=1, \ldots, m$, we have

$$
\begin{aligned}
\|( & (\Gamma u)(t)-(\Gamma v)(t) \| \\
= & \| T\left(t-t_{i}\right) g_{i}\left(s_{i}, u\left(s_{i}\right)\right)+\int_{s_{i}}^{t} T(t-s)(f(s, u(s), u(\rho(s)))+B(s) c(s)) d s \\
& \quad-T\left(t-t_{i}\right) g_{i}\left(s_{i}, v\left(s_{i}\right)\right)-\int_{s_{i}}^{t} T(t-s)(f(s, v(s), v(\rho(s)))+B(s) c(s)) d s \| \\
& \leq M\left[C_{g_{i}}+\left(C_{f}+L_{f}\right)\left(t_{i+1}-s_{i}\right)\right]\|u-v\|_{\mathcal{P C}} \\
\leq & M \max _{1 \leq i \leq m}\left\{C_{g_{i}}+\left(C_{f}+L_{f}\right)\left(t_{i+1}-s_{i}\right)\right\}\|u-v\|_{\mathcal{P C}} \\
\leq & \lambda\|u-v\|_{\mathcal{P C}} .
\end{aligned}
$$

Therefore, we obtain

$$
\|\Gamma u-\Gamma v\|_{\mathcal{P C}} \leq \lambda\|u-v\|_{\mathcal{P C}}, \quad \forall u, v \in \mathcal{P C}(I, X) .
$$

Finally, we find that $\Gamma$ is a contraction mapping on $\mathcal{P C}(I, X)$, and there exists a unique $u \in \mathcal{P C}(I, X)$ such that $\Gamma u=u$.

So we conclude that $u$ is the unique mild solution of (IEE).

By using Krasnoselskii's fixed point theorem, we also obtain the existence of a mild solution.

Theorem 3 Let assumptions $\left(\mathrm{H}_{0}\right),\left(\mathrm{H}_{1}\right),\left(\mathrm{H}_{3}\right)$, and $\left(\mathrm{H}_{5}\right)$ be satisfied. Suppose, in addition, that the semigroup $\{T(t), t \geq 0\}$ is compact and

$$
\begin{aligned}
& \alpha:=\max \left\{L M\left(M\left(a-s_{m}\right)+t_{1}\right), L M\left(t_{i+1}-s_{i}\right)\right\}<\frac{1}{2}, \quad i=1, \ldots, m, \\
& \beta:=\max \left\{M^{2} C_{g_{m}}, M C_{g_{i}}\right\}<1 .
\end{aligned}
$$

Then the problem (IEE) has at least one mild solution.

Proof Let $N=\max \left(N_{1}, N_{2}, \ldots, N_{m}\right)$ and $B_{r}=\left\{u \in \mathcal{P C}(I, X):\|u\|_{\mathcal{P C}}<r\right\}$ the ball with radius $r>0$.

Here

$$
r \geq \max \left\{\gamma_{1}, \gamma_{2}, M N\right\}
$$


with

$$
\gamma_{1}=\frac{M^{2} N_{m}+\left(M^{2}+M\right)\|B c\|_{L^{1}}+L M\left(M\left(a-s_{m}\right)+t_{1}\right)}{1-2 \alpha}
$$

and

$$
\gamma_{2}=\frac{M N_{i}+M\|B c\|_{L^{1}}+M L\left(t_{i+1}-s_{i}\right)}{1-2 \alpha} .
$$

We introduce the decomposition $\Gamma=\Gamma_{1}+\Gamma_{2}$, where

$$
\left(\Gamma_{1} u\right)(t)= \begin{cases}T(t) T\left(a-t_{m}\right) g_{m}\left(s_{m}, u\left(s_{m}\right)\right), & t \in\left[0, t_{1}\right], \\ T\left(t-t_{i}\right) g_{i}(t, u(t)), & t \in\left(t_{i}, s_{i}\right], i=1,2, \ldots, m, \\ T\left(t-t_{i}\right) g_{i}\left(s_{i}, u\left(s_{i}\right)\right), & t \in\left(s_{i}, t_{i+1}\right], i=1,2, \ldots, m\end{cases}
$$

and

$$
\left(\Gamma_{2} u\right)(t)= \begin{cases}T(t) \int_{s_{\eta}}^{a} T(a-s)(f(s, u(s), u(\rho(s)))+B(s) c(s)) d s & \\ \quad+\int_{0}^{t} T(t-s)(f(s, u(s), u(\rho(s)))+B(s) c(s)) d s, & t \in\left[0, t_{1}\right], \\ 0, & t \in\left(t_{i}, s_{i}\right], i=1,2, \ldots, m, \\ \int_{s_{i}}^{t} T(t-s)(f(s, u(s), u(\rho(s)))+B(s) c(s)) d s, & t \in\left(s_{i}, t_{i+1}\right], i=1,2, \ldots, m .\end{cases}
$$

We distinguish in the proof several steps.

Step 1. We prove that $\Gamma u=\Gamma_{1} u+\Gamma_{2} u \in B_{r}$ for all $u \in B_{r}$. Indeed:

Case 1. For $t \in\left[0, t_{1}\right]$, we have

$$
\begin{aligned}
&\left\|\left(\Gamma_{1} u+\Gamma_{2} u\right)(t)\right\| \\
& \leq\|T(t)\|\left\|T\left(a-t_{m}\right) g_{m}\left(s_{m}, u\left(s_{m}\right)\right)\right\| \\
& \quad+\|T(t)\| \int_{s_{m}}^{a}\|T(a-s)\|(\|f(s, u(s), u(\rho(s)))\|+\|B(s) c(s)\|) d s \\
& \quad+\int_{0}^{t}\|T(t-s)\|(\|f(s, u(s), u(\rho(s)))\|+\|B(s) c(s)\|) d s \\
& \leq M^{2} N_{m}+L M^{2} \int_{s_{m}}^{a}\left(1+\|u(s)\|^{\mu}+\|u(\rho(s))\|^{\nu}\right) d s+\left(M^{2}+M\right)\|B c\|_{L^{1}} \\
& \quad+L M \int_{0}^{t}\left(1+\|u(s)\|^{\mu}+\|u(\rho(s))\|^{\nu}\right) d s \\
& \leq M^{2} N_{m}+\left(M^{2}+M\right)\|B c\|_{L^{1}}+L M^{2}(1+2 r)\left(a-s_{m}\right)+L M(1+2 r) t_{1} \\
&=M^{2} N_{m}+\left(M^{2}+M\right)\|B c\|_{L^{1}}+L M\left(M\left(a-s_{m}\right)+t_{1}\right)+2 r L M\left(M\left(a-s_{m}\right)+t_{1}\right) \\
& \leq r(1-2 \alpha)+2 r \alpha=r .
\end{aligned}
$$

Case 2. For $t \in\left(t_{i}, s_{i}\right], i=1, \ldots, m$, we have

$$
\begin{aligned}
\left\|\left(\Gamma_{1} u+\Gamma_{2} u\right)(t)\right\| & \leq\left\|T\left(t-t_{i}\right)\right\|\left\|g_{i}(t, u(t))\right\| \\
& \leq M N_{i} \leq M N \leq r .
\end{aligned}
$$


Case 3. For $t \in\left(s_{i}, t_{i+1}\right], i=1, \ldots, m$, we have

$$
\begin{aligned}
\left\|\left(\Gamma_{1} u+\Gamma_{2} u\right)(t)\right\| \leq & \left\|T\left(t-t_{i}\right)\right\|\left\|g_{i}\left(s_{i}, u\left(s_{i}\right)\right)\right\| \\
& +\int_{s_{i}}^{t}\|T(t-s)\|(\|f(s, u(s), u(\rho(s)))\|+\|B(s) c(s)\|) d s \\
\leq & M N_{i}+L M(1+2 r)\left(t_{i+1}-s_{i}\right)+M\|B c\|_{L^{1}} \\
& =M N_{i}+M\|B c\|_{L^{1}}+L M\left(t_{i+1}-s_{i}\right)+2 r L M\left(t_{i+1}-s_{i}\right) \\
\leq & r(1-2 \alpha)+2 r \alpha=r .
\end{aligned}
$$

Then we deduce that $\Gamma_{1} u+\Gamma_{2} u \in B_{r}$.

Step 2. $\Gamma_{1}$ is contraction on $B_{r}$. Let $u, v \in B_{r}$.

Case 1 . For $t \in\left[0, t_{1}\right]$, we have

$$
\begin{aligned}
\left\|\left(\Gamma_{1} u\right)(t)-\left(\Gamma_{1} v\right)(t)\right\| & \leq\|T(t)\|\left\|T\left(a-t_{m}\right)\right\|\left\|g_{m}\left(s_{m}, u\left(s_{m}\right)\right)-g_{m}\left(s_{m}, v\left(s_{m}\right)\right)\right\| \\
& \leq M^{2} C_{g_{m}}\left\|u\left(s_{m}\right)-v\left(s_{m}\right)\right\| \\
& \leq M^{2} C_{g_{m}}\|u-v\|_{\mathcal{P C}} \\
& \leq \beta\|u-v\|_{\mathcal{P C}} .
\end{aligned}
$$

Case 2. For $t \in\left(t_{i}, s_{i}\right], i=1, \ldots, m$, we have

$$
\begin{aligned}
\left\|\left(\Gamma_{1} u\right)(t)-\left(\Gamma_{1} v\right)(t)\right\| & \leq\left\|T\left(t-t_{i}\right)\right\|\left\|g_{i}(t, u(t))-g_{i}(t, v(t))\right\| \\
& \leq M C_{g_{m}}\|u-v\|_{\mathcal{P C}} \\
& \leq \beta\|u-v\|_{\mathcal{P C}} .
\end{aligned}
$$

Case 3. For $t \in\left(s_{i}, t_{i+1}\right], i=1, \ldots, m$, we have

$$
\begin{aligned}
\left\|\left(\Gamma_{1} u\right)(t)-\left(\Gamma_{1} v\right)(t)\right\| & \leq\left\|T\left(t-t_{i}\right)\right\|\left\|g_{i}\left(s_{i}, u\left(s_{i}\right)\right)-g_{i}\left(s_{i}, v\left(s_{i}\right)\right)\right\| \\
& \leq M C_{g_{m}}\|u-v\|_{\mathcal{P C}} \\
& \leq \beta\|u-v\|_{\mathcal{P C}} .
\end{aligned}
$$

This implies that $\Gamma_{1}$ is a contraction.

Step 3. $\Gamma_{2}$ is continuous.

Let $\left(u_{n}\right)_{n \geq 0}$ be a sequence such that $\lim _{n \rightarrow+\infty}\left\|u_{n}-u\right\|_{\mathcal{P C}}=0$.

Case 1 . For $t \in\left[0, t_{1}\right]$, we have

$$
\begin{gathered}
\left\|\left(\Gamma_{2} u_{n}\right)(t)-\left(\Gamma_{2} u\right)(t)\right\| \\
\leq\|T(t)\| \int_{s_{m}}^{a}\|T(a-s)\|\left\|f\left(s, u_{n}(s), u_{n}(\rho(s))\right)-f(s, u(s), u(\rho(s)))\right\| d s \\
\quad+\int_{0}^{t}\|T(t-s)\|\left\|f\left(s, u_{n}(s), u_{n}(\rho(s))\right)-f(s, u(s), u(\rho(s)))\right\| d s \\
\leq M^{2}\left(a-s_{m}\right)\left\|f\left(\cdot, u_{n}(\cdot), u_{n}(\rho(\cdot))\right)-f(\cdot, u(\cdot), u(\rho(\cdot)))\right\|_{\mathcal{P C}}
\end{gathered}
$$




$$
\begin{aligned}
& +M t_{1}\left\|f\left(\cdot, u_{n}(\cdot), u_{n}(\rho(\cdot))\right)-f(\cdot, u(\cdot), u(\rho(\cdot)))\right\|_{\mathcal{P C}} \\
= & M\left[M\left(a-s_{m}\right)+t_{1}\right]\left\|f\left(\cdot, u_{n}(\cdot), u_{n}(\rho(\cdot))\right)-f(\cdot, u(\cdot), u(\rho(\cdot)))\right\|_{\mathcal{P C}} .
\end{aligned}
$$

Case 2. For $t \in\left(t_{i}, s_{i}\right], i=1, \ldots, m$, we have

$$
\left\|\left(\Gamma_{2} u_{n}\right)(t)-\left(\Gamma_{2} u\right)(t)\right\|=0 .
$$

Case 3. For $t \in\left(s_{i}, t_{i+1}\right], i=1, \ldots, m$, we have

$$
\begin{aligned}
\left\|\left(\Gamma_{2} u_{n}\right)(t)-\left(\Gamma_{2} u\right)(t)\right\| & \leq \int_{s_{i}}^{t}\|T(t-s)\|\left\|f\left(s, u_{n}(s), u_{n}(\rho(s))\right)-f(s, u(s), u(\rho(s)))\right\| d s \\
& =M\left(t_{i+1}-s_{i}\right)\left\|f\left(\cdot, u_{n}(\cdot), u_{n}(\rho(\cdot))\right)-f(\cdot, u(\cdot), u(\rho(\cdot)))\right\|_{\mathcal{P C}}
\end{aligned}
$$

This implies that $\lim _{n \rightarrow+\infty}\left\|\Gamma_{2} u_{n}-\Gamma_{2} u\right\|_{\mathcal{P C}}=0$, then we deduce that $\Gamma_{2}$ is continuous.

Step $4 . \Gamma_{2}$ is compact.

1. We have $\Gamma_{2} B_{r} \subseteq B_{r}$, then $\Gamma_{2}$ is uniformly bounded on $B_{r}$.

2. For $u \in B_{r}$, we have the following.

Case 1. For $0 \leq l_{1}<l_{2} \leq t_{1}$, we have

$$
\begin{aligned}
&\left\|\left(\Gamma_{2} u\right)\left(l_{2}\right)-\left(\Gamma_{2} u\right)\left(l_{1}\right)\right\| \\
& \leq \| T\left(l_{2}\right)-T\left(l_{1}\right)\left\|\int_{s_{m}}^{a}\right\| T(a-s) \|(\|f(s, u(s), u(\rho(s)))\|+\|B(s) c(s)\|) d s \\
& \quad+\int_{0}^{l_{1}}\left\|T\left(l_{2}-s\right)-T\left(l_{1}-s\right)\right\|(\|f(s, u(s), u(\rho(s)))\|+\|B(s) c(s)\|) d s \\
& \quad+\int_{l_{1}}^{l_{2}}\left\|T\left(l_{2}-s\right)\right\|(\|f(s, u(s), u(\rho(s)))\|+\|B(s) c(s)\|) d s \\
& \leq \quad M^{2}\left(L(1+2 r)\left(a-s_{m}\right)+\|B c\|_{L^{1}}\right)\left\|T\left(l_{2}-l_{1}\right)-I\right\| \\
& \quad+M\left(L(1+2 r) t_{1}+\|B c\|_{L^{1}}\right)\left\|T\left(l_{2}-l_{1}\right)-I\right\| \\
& \quad+L M(1+2 r)\left(l_{2}-l_{1}\right)+M \int_{l_{1}}^{l_{2}}\|B(s) c(s)\| d s \\
&=\left(L M(1+2 r)\left[M\left(a-s_{m}\right)+t_{1}\right]+\left(M^{2}+M\right)\|B c\|_{L^{1}}\right)\left\|T\left(l_{2}-l_{1}\right)-I\right\| \\
& \quad+L M(1+2 r)\left(l_{2}-l_{1}\right)+M \int_{l_{1}}^{l_{2}}\|B(s) c(s)\| d s \rightarrow 0 \quad \text { as } l_{2} \rightarrow l_{1} .
\end{aligned}
$$

Since $\{T(t), t \geq 0\}$ is compact, $\left\|T\left(l_{2}-l_{1}\right)-I\right\| \rightarrow 0$ as $l_{2} \rightarrow l_{1}$.

Case 2. For $t_{i} \leq l_{1}<l_{2} \leq s_{i}, i=1, \ldots, m$, we have

$$
\left\|\left(\Gamma_{2} u\right)\left(l_{2}\right)-\left(\Gamma_{2} u\right)\left(l_{1}\right)\right\|=0 .
$$

Case 3. For $s_{i} \leq l_{1}<l_{2} \leq t_{i+1}, i=1, \ldots, m$, we have

$$
\begin{aligned}
& \left\|\left(\Gamma_{2} u\right)\left(l_{2}\right)-\left(\Gamma_{2} u\right)\left(l_{1}\right)\right\| \\
& \quad=\| \int_{s_{i}}^{l_{2}} T\left(l_{2}-s\right)(f(s, u(s), u(\rho(s)))+B(s) c(s)) d s
\end{aligned}
$$




$$
\begin{aligned}
& -\int_{s_{i}}^{l_{1}} T\left(l_{1}-s\right)(f(s, u(s), u(\rho(s)))+B(s) c(s)) d s \| \\
\leq & \int_{l_{1}}^{l_{2}}\left\|T\left(l_{2}-s\right)\right\|(\|f(s, u(s), u(\rho(s)))\|+\|B(s) c(s)\|) d s \\
& +\int_{s_{i}}^{l_{1}}\left\|T\left(l_{1}-s\right)\right\|\left\|T\left(l_{2}-l_{1}\right)-I\right\|(\|f(s, u(s), u(\rho(s)))\|+\|B(s) c(s)\|) d s \\
\leq & L M(1+2 r)\left(l_{2}-l_{1}\right)+M \int_{l_{1}}^{l_{2}}\|B(s) c(s)\| d s \\
& +M\left(L(1+2 r)\left(t_{i+1}-s_{i}\right)+\|B c\|_{L^{1}}\right)\left\|T\left(l_{2}-l_{1}\right)-I\right\| \rightarrow 0 \quad \text { as } l_{2} \rightarrow l_{1} .
\end{aligned}
$$

This permits us to conclude that $\Gamma_{2}$ is equicontinuous.

We have $\Gamma_{2} B_{r} \subseteq B_{r}$, let $\Theta:=\Gamma_{2} B_{r}, \Theta(t):=\Gamma_{2} B_{r}(t)=\left\{\left(\Gamma_{2} u\right)(t): u \in B_{r}\right\}$ for $t \in[0, a]$.

3. $\Theta(t)$ is relatively compact. Indeed:

$T(t)$ is compact, hence

$$
\Theta(0)=\left\{\int_{s_{m}}^{a} T(a-s)(f(s, u(s), u(\rho(s)))+B(s) c(s)) d s\right\},
$$

is relatively compact. For $0<\varepsilon<t \leq a$, define

$$
\Theta_{\varepsilon}(t):=\Gamma_{2}^{\varepsilon} B_{r}(t)=\left\{T(\varepsilon)\left(\Gamma_{2} u\right)(t-\varepsilon): u \in B_{r}\right\} .
$$

Clearly, $\Theta_{\varepsilon}(t)$ is relatively compact for $t \in(\varepsilon, a]$, since $T(t)$ is compact.

Case 1. For $t \in\left(0, t_{1}\right]$, we have

$$
\begin{aligned}
\Theta_{\varepsilon}(t):= & \left(\Gamma_{2}^{\varepsilon} u\right)(t)=T(\varepsilon)\left(\Gamma_{2} u\right)(t-\varepsilon) \\
= & \left\{T(\varepsilon) T(t-\varepsilon)\left[\int_{s_{m}}^{a} T(a-s)(f(s, u(s), u(\rho(s)))+B(s) c(s)) d s\right]\right. \\
& \left.+T(\varepsilon) \int_{0}^{t-\varepsilon} T(t-\varepsilon-s)(f(s, u(s), u(\rho(s)))+B(s) c(s)) d s: u \in B_{r}\right\} \\
= & \left\{T(t)\left[\int_{s_{m}}^{a} T(a-s)(f(s, u(s), u(\rho(s)))+B(s) c(s)) d s\right]\right. \\
& \left.+\int_{0}^{t-\varepsilon} T(t-s)(f(s, u(s), u(\rho(s)))+B(s) c(s)) d s: u \in B_{r}\right\},
\end{aligned}
$$

and we get

$$
\begin{aligned}
\|( & \left.\Gamma_{2} u\right)(t)-\left(\Gamma_{2}^{\varepsilon} u\right)(t) \| \\
= & \| \int_{0}^{t} T(t-s)(f(s, u(s), u(\rho(s)))+B(s) c(s)) d s \\
& \quad-\int_{0}^{t-\varepsilon} T(t-s)(f(s, u(s), u(\rho(s)))+B(s) c(s)) d s \| \\
\leq & \int_{t-\varepsilon}^{t}\|T(t-s)\|(\|f(s, u(s), u(\rho(s)))\|+\|B(s) c(s)\|) d s \\
\leq & L M(1+2 r) \varepsilon+M \int_{t-\varepsilon}^{t}\|B(s) c(s)\| d s \rightarrow 0 \quad \text { as } \varepsilon \rightarrow 0 .
\end{aligned}
$$


Case 2. For $t \in\left(t_{i}, s_{i}\right], i=1, \ldots, m$, we have

$$
\Theta_{\varepsilon}(t):=\left\{0, u \in B_{r}\right\}
$$

in this case $\left\|\left(\Gamma_{2} u\right)(t)-\left(\Gamma_{2}^{\varepsilon} u\right)(t)\right\|=0$.

Case 3. For $t \in\left(s_{i}, t_{i+1}\right], i=1, \ldots, m$, we have

$$
\begin{aligned}
\Theta_{\varepsilon}(t) & :=\left(\Gamma_{2}^{\varepsilon} u\right)(t) \\
& =\left\{T(\varepsilon) \int_{s_{i}}^{t-\varepsilon} T(t-\varepsilon-s)(f(s, u(s), u(\rho(s)))+B(s) c(s)) d s: u \in B_{r}\right\} \\
& =\left\{\int_{s_{i}}^{t-\varepsilon} T(t-s)(f(s, u(s), u(\rho(s)))+B(s) c(s)) d s: u \in B_{r}\right\}
\end{aligned}
$$

and we get

$$
\begin{aligned}
\|( & \left.\Gamma_{2} u\right)(t)-\left(\Gamma_{2}^{\varepsilon} u\right)(t) \| \\
= & \| \int_{s_{i}}^{t} T(t-s)(f(s, u(s), u(\rho(s)))+B(s) c(s)) d s \\
& \quad-\int_{s_{i}}^{t-\varepsilon} T(t-s)(f(s, u(s), u(\rho(s)))+B(s) c(s)) d s \| \\
\leq & \int_{t-\varepsilon}^{t}\|T(t-s)\|(\|f(s, u(s), u(\rho(s)))\|+\|B(s) c(s)\|) d s \\
\leq & L M(1+2 r) \varepsilon+M \int_{t-\varepsilon}^{t}\|B(s) c(s)\| d s \rightarrow 0 \quad \text { as } \varepsilon \rightarrow 0 .
\end{aligned}
$$

Now, from the Arzela-Ascoli theorem we can conclude that $\Gamma_{2}: B_{r} \longrightarrow B_{r}$ is completely continuous. The existence of a mild solution for (IEE) is now a consequence of Krasnoselskii's fixed point theorem.

\section{Examples}

In this section, we give examples to illustrate our abstract results in the previous section.

Let $X=L^{2}(0,1), I=[0,3], 0=t_{0}=s_{0}, t_{1}=1, s_{1}=2$, and $a=3$. Define $A v=\frac{\partial^{2}}{\partial^{2} x} v$ for

$$
v \in D(A)=\left\{v \in X: \frac{\partial v}{\partial x}, \frac{\partial^{2} v}{\partial^{2} x} \in X, v(0)=v(1)=0\right\} .
$$

Then $A$ is the infinitesimal generator of a strongly continuous semigroup $\{T(t), t \geq 0\}$ on $X$. In addition $T(t)$ is compact and $\|T(t)\| \leq 1$, for all $t \geq 0$.

\section{Example 1 Consider}

$$
\left\{\begin{array}{l}
\frac{\partial}{\partial t} u(t, x)=\frac{\partial^{2}}{\partial{ }^{2} x} u(t, x)+\frac{1}{12} \cos \left(u(t, x)+u\left(t^{2}, x\right)\right)+c(t, x), \quad x \in(0,1), t \in[0,1) \cup(2,3] \\
\frac{\partial}{\partial x} u(t, 0)=\frac{\partial}{\partial x} u(t, 1)=0, \quad t \in[0,1) \cup(2,3] \\
u(0, x)=u(3, x), \quad x \in(0,1), \\
u(t, x)=T(t-1) \frac{1}{4} \sin (u(t, x)), \quad x \in(0,1), t \in(1,2]
\end{array}\right.
$$


Denote $v(t)(x)=u(t, x)$ and $B(t) c(t)(x)=c(t, x)$, this problem can be abstracted into

$$
\left\{\begin{array}{l}
v^{\prime}(t)=A v(t)+f(t, v(t), v(\rho(t)))+B(t) c(t), \quad t \in\left[s_{0}, t_{1}\right) \cup\left(s_{1}, a\right] \\
v(t)=T\left(t-t_{i}\right) g_{1}(t, v(t)), \quad t \in\left(t_{1}, s_{1}\right] \\
v(0)=v(a) \in X
\end{array}\right.
$$

where $\rho(t)=t^{2}, f(t, v(t), v(\rho(t)))(x)=\frac{1}{12} \cos \left(v(t)(x)+v\left(t^{2}\right)(x)\right)$ and

$$
g_{1}(t, v(t))(x)=\frac{1}{4} \sin (v(t)(x))
$$

In this case, we have $M=1, C_{f}=L_{f}=\frac{1}{12}, C_{g_{1}}=\frac{1}{4}$, and

$$
\lambda=M\left[M C_{g_{1}}+\left(C_{f}+L_{f}\right)\left(a-s_{1}\right)+\left(C_{f}+L_{f}\right) t_{1}\right]=\frac{7}{12}<1 .
$$

This implies that all assumptions in Theorem 2 are satisfied. Then there exists a unique mild solution for this problem.

Example 2 Consider

$$
\left\{\begin{array}{l}
\frac{\partial}{\partial t} u(t, x)=\frac{\partial^{2}}{\partial^{2} x} u(t, x)+\frac{1}{8 e^{t}} \frac{\left|u(t, x)+u\left(t^{2}, x\right)\right|}{1+\left|u(t, x)+u\left(t^{2}, x\right)\right|}+c(t, x), \quad x \in(0,1), t \in[0,1) \cup(2,3], \\
\frac{\partial}{\partial x} u(t, 0)=\frac{\partial}{\partial x} u(t, 1)=0, \quad t \in[0,1) \cup(2,3], \\
u(0, x)=u(3, x), \quad x \in(0,1), \\
u(t, x)=T(t-1) \frac{1}{8 e^{t-1}} \frac{|u(t, x)|}{1+|u(t, x)|}, \quad x \in(0,1), t \in(1,2] .
\end{array}\right.
$$

This problem can be abstracted into (1), with $\rho(t)=t^{2}$,

$$
\begin{aligned}
& f(t, v(t), v(\rho(t)))(x)=\frac{1}{8 e^{t}} \frac{\left|v(t)(x)+v\left(t^{2}\right)(x)\right|}{1+\left|v(t)(x)+v\left(t^{2}\right)(x)\right|}, \\
& g_{1}(t, v(t))(x)=\frac{1}{8 e^{t-1}} \frac{|v(t)(x)|}{1+|v(t)(x)|}
\end{aligned}
$$

and $B(t) c(t)(x)=c(t, x)$.

In this case, we have $L=\frac{1}{8}, C_{g_{1}}=\frac{1}{8}, \alpha=\frac{1}{4}<\frac{1}{2}$, and $\beta=\frac{1}{8}<1$.

This implies that all assumptions in Theorem 3 are satisfied. Then this problem has at least one mild solution.

\section{Conclusion}

In order to describe the evolution of the temperature using a control, we consider periodic boundary value problems for controlled nonlinear impulsive evolution equations. By using operator semigroup theory, impulsive conditions, and fixed point methods, we overcome some difficulties from the proof of equicontinuity and compactness and obtain new existence results. In addition, future work includes expanding the idea signalized in this work and introducing observability. This is a fertile field with vast research projects, which can lead to numerous theories and applications. We plan to devote significant attention to this field of research. 


\section{Competing interests}

The authors declare that they have no competing interests.

\section{Authors' contributions}

All authors contributed equally to the writing of this paper. All authors read and approved the final manuscript.

\section{Acknowledgements}

The authors express their sincere thanks to the anonymous referees for numerous helpful and constructive suggestions which have improved the manuscript.

\section{Received: 8 February 2016 Accepted: 18 October 2016 Published online: 11 November 2016}

\section{References}

1. Feckan, M, Wang, JR: A general class of impulsive evolution equations. Topol. Methods Nonlinear Anal. 46, 915-934 (2015)

2. Liu, S, Wang, JR, Wei, W: A study on iterative learning control for impulsive differential equations. Commun. Nonlinear Sci. Numer. Simul. 24, 4-10 (2015)

3. Yu, X, Wang, JR: Periodic boundary value problems for nonlinear impulsive evolution equations on Banach spaces. Commun. Nonlinear Sci. Numer. Simul. 22, 980-989 (2015)

4. Wang, JR, Feckan, M, Zhou, Y: Noninstantaneous impulsive models for studying periodic evolution processes in pharmacotherapy. In: Luo, A, Merdan, H (eds.) Mathematical Modeling and Applications in Nonlinear Dynamics. Nonlinear Systems and Complexity, vol. 14, pp. 87-107. Springer, Cham (2016)

5. Wang, JR, Zhang, Y: On the concept and existence of solutions for fractional impulsive systems with Hadamard derivatives. Appl. Math. Lett. 39, 85-90 (2015)

6. Wang, JR, Ibrahim, AG, Feckan, M: Nonlocal impulsive fractional differential inclusions with fractional sectorial operators on Banach spaces. Appl. Math. Comput. 257, 103-118 (2015)

7. Wang, JR, Feckan, M, Zhou, Y: Ulam's type stability of impulsive ordinary differential equations. J. Math. Anal. Appl. $395,258-264(2012)$

8. Wang, JR, Feckan, M, Zhou, Y: On the new concept of solutions and existence results for impulsive fractional evolution equations. Dyn. Partial Differ. Equ. 8(4), 345-361 (2011)

9. Wang, JR, Zhou, Y, Lin, Z: On a new class of impulsive fractional differential equations. Appl. Math. Comput. 242 649-657 (2014)

10. Wang, JR, Feckan, M, Zhou, Y: Relaxed controls for nonlinear fractional impulsive evolution equations. J. Optim Theory Appl. 156, 13-32 (2013)

11. Wang, JR, Zhou, Y, Medved, M: Picard and weakly Picard operators technique for nonlinear differential equations in Banach spaces. J. Math. Anal. Appl. 389, 261-274 (2012)

12. Bainov, DD, Simeonov, PS: Impulsive Differential Equations: Periodic Solutions and Applications. Longman, New York (1993)

13. Samoilenko, AM, Perestyuk, NA: Impulsive Differential Equations. World Scientific, Singapore (1995)

14. Liu, JH: Nonlinear impulsive evolution equations. Dyn. Contin. Discrete Impuls. Syst. 6, 77-85 (1999)

15. Bainov, D, Kamont, Z, Minchev, E: Periodic boundary value problem for impulsive hyperbolic partial differential equations of first order. Appl. Math. Comput. 68(2-3), 95-104 (1995)

16. Hernandez, E, O’Regan, D: On a new class of abstract impulsive differential equations. Proc. Am. Math. Soc. 141(5), 1641-1649 (2013)

17. Hernandez, ME, Tanaka Aki, SM, Henriquez, H: Global solutions for impulsive abstract partial differential equations. Comput. Math. Appl. 56, 1206-1215 (2008)

18. Pierri, M, O'Regan, D, Rolnik, V: Existence of solutions for semi-linear abstract differential equations with not instantaneous impulses. Appl. Math. Comput. 219,6743-6749 (2013)

19. Ahmed, NU, Teo, KL, Hou, SH: Nonlinear impulsive systems on infinite dimensional spaces. Nonlinear Anal. 54 907-925 (2003)

20. Ahmed, NU, Xiang, X: Nonlinear uncertain systems and necessary conditions of optimality. SIAM J. Control Optim. 35 1755-1772 (1997)

21. Li, X, Yong, J: Optimal Control Theory for Infinite Dimensional Systems. Birkhäuser, Basel (1995)

22. Wei, W, Xiang, X, Peng, Y: Nonlinear impulsive integro-differential equations of mixed type and optimal controls. Optimization 55, 141-156 (2006)

23. Pongchalee, $P$, Sattayatham, $P, X i a n g, X$ : Relaxation of nonlinear impulsive controlled systems on Banach spaces. Nonlinear Anal. 68, 1570-1580 (2008)

24. Zhu, L, Huang, Q: Nonlinear impulsive evolution equations with nonlocal conditions and optimal controls. Adv. Differ. Equ. 2015, 378 (2015)

25. Lakshmikantham, V, Bainov, DD, Simeonov, PS: Theory of Impulsive Differential Equations. World Scientific, Singapore (1989) 